ISSN: 0212-5374

DOI: http://dx.doi.org/10.14201/et201432197116

\title{
ANÁLISIS DE PROCESOS COGNITIVOS \\ EN LA ESCRITURA DE ALUMNOS SORDOS Y OYENTES DE ESPAÑA E ITALIA
}

\author{
Analysis of the writing processes in deaf and hearing \\ students from Spain and Italy
}

\section{Analyse des processus cognitifs en écriture des élèves sourds et entendants en Espagne et en Italie}

Rafaela GUTIÉRREZ CÁCERES* y Antonio LUQUE DE LA ROSA**

Universidad de Almería. Correo-e: rcaceres@ual.es;

Recibido: 20-05-2013; Aceptado: 11-11-2013; Publicado: 30-03-2014

BIBLID [0212-5374 (2014) 32, 1; 97-116]

Ref. Bibl. RAFAELA GUTIÉRREZ CÁCERES y ANTONIO LUQUE DE LA ROSA. Análisis de procesos cognitivos en la escritura de alumnos sordos y oyentes de España e Italia. Enseñanza \& Teaching, 32, 1-2014, 97-116.

RESUMEN: En este artículo se muestran algunos resultados principales de una investigación centrada en el análisis de los procesos cognitivos y metacognitivos implicados en la composición escrita por parte del alumnado sordo y oyente procedente de dos contextos escolares (España e Italia).

Los sujetos participantes en la investigación fueron 84 alumnos: 72 oyentes y 12 sordos; 44 del contexto español y 40 del italiano. El proceso de recogida de datos ha consistido en la aplicación de un cuestionario adaptado sobre la autoevaluación de los procesos escritores. En cuanto al procedimiento de análisis, se ha llevado a cabo una comparación y confrontación de las habilidades cognitivas del alumnado en función del estatus "sordo/oyente" así como del contexto escolar de procedencia.

En general se ha encontrado que los alumnos sordos y oyentes escolarizados en los centros español e italiano coinciden en afirmar que realizan los procesos 
RAFAELA GUTIÉRREZ CÁCERES Y ANTONIO LUQUE DE LA ROSA

ANÁLISIS DE PROCESOS COGNITIVOS EN LA ESCRITURA DE ALUMNOS SORDOS

Y OYENTES DE ESPAÑA E ITALIA

referidos a la planificación, transcripción, revisión y metacognición necesarios para desarrollar la calidad de los textos escritos. No obstante, hay que señalar que existen algunos aspectos significativos dada la variabilidad en las respuestas a cada ítem del cuestionario y la diversidad entre el alumnado. Ante la escasez de este tipo de investigaciones se requiere seguir emprendiendo estudios centrados en el análisis de los componentes cognitivos de la composición escrita en alumnos sordos, en función de características individuales y contextuales.

Palabras clave: escritura, procesos cognitivos, metacognición, discapacidad auditiva, contexto escolar, bilingüismo.

SUMMARY: In this paper we present some of the most relevant results from research that focused on the analysis of cognitive and metacognitive processes involved in written composition by the deaf and hearing students from two school contexts (Spain and Italy).

Research participants were 84 students: 72 hearing and 12 deaf; 44 from Spanish context and 40 from Italy. The data collection process consisted of the application of an adapted questionnaire on self-evaluation of the processes in writing. Regarding the procedure of analysis has been carried out a comparison and confrontation of cognitive skills of students in terms of status "deaf/hearing" and school context.

Generally found that deaf and hearing students integrated in schools from Spain and Italy saying that perform the processes related to planning, transcription, review and metacognition needed to develop the quality of written texts. However, it should be noted that there are some significant aspects given the variety in the answers to each item and diversity among students. In the lack of this type of research is required continue to undertake studies focusing on the analysis of the cognitive components of writing in deaf students, depending on individual and contextual characteristics.

Key words: writing, cognitive processes, metacognition, hearing impaired, school context, bilingualism.

RÉSUMÉ: Dans cet article, nous présentons les principaux résultats d'une recherche sur l'analyse des processus cognitifs et métacognitifs impliqués dans la composition écrite par les élèves sourds et entendants de deux contextes scolaires (Espagne et Italie).

Les sujets participant à l'étude étaient 84 élèves: 72 entendants et 12 sourds, 44 élèves du contexte espagnol et 40 du contexte italien. Dans la collecte des données a été effectuée l'application d'un questionnaire adapté sur l'auto-évaluation des processus dans le composition écrite. En ce qui concerne la procédure d'analyse, a été effectué une comparaison et confrontation des compétences cognitives des élèves en fonction du statut "sourd/entendant" et du contexte scolaire.

Dans l'ensemble, nous avons constaté que les étudiants sourds et entendants scolarisés dans les centres espagnols et italiens affirment qu'ils exécutent les processus liés à la planification, la transcription, la revision et la métacognition, qui sont 
nécessaires pour améliorer la qualité des textes écrits. Cependant, il faut considérer des égards compte tenu de la variabilité dans les réponses à chaque item du questionnaire et de la diversité parmi les étudiants. Face à l'échec de ce type de recherche, il est nécessaire de réaliser des études sur l'analyse des processus cognitifs dans la composition écrite chez les élèves sourds, en fonction des caractéristiques individuelles et contextuelles.

Mots clés: écriture, processus cognitifs, métacognition, surdité, contexte scolaire, bilinguisme.

\section{INTRODUCCIÓN}

«El aprendizaje de la lengua escrita es un objetivo básico y prioritario para el alumnado sordo, por cuanto esta competencia comunicativa favorece el acceso a otros aprendizajes" (Carrillo y Domínguez, 2010; Fernández y Pertusa, 2007; Jáudenes, 2009). Siendo así, en este caso concreto, la escritura una habilidad cognitiva compleja que se desarrolla en una situación comunicativa y social (Arroyo y Salvador, 2009; MacArthur, Graham y Fitzgerald, 2006).

Siguiendo los modelos teóricos (Flower y Hayes, 1981, 1984; Hayes, 1996), la composición escrita supone, más allá de la traducción físico-motora de los signos convencionales, la interacción y recursividad de los procesos cognitivos de planificación, transcripción y revisión, bajo la supervisión de las habilidades metacognitivas.

El proceso de planificación hace referencia a la realización de un plan previo de la composición representando mentalmente las ideas, en función de las exigencias del tema, del destinatario, de la finalidad comunicativa y de la organización del texto (Berninger y Whitaker, 1993; Berninger, Fuller y Whitaker, 1996). A su vez, este proceso incluye varios subprocesos cognitivos: génesis de ideas; organización o estructuración de la información recuperada y seleccionada; selección de objetivos que guían y controlan el acto y el producto de la composición.

La transcripción consiste en estructurar las ideas previamente planificadas en un texto escrito, atendiendo a los aspectos tanto gramaticales como discursivos (ortografía, puntuación, léxico, sintaxis, cohesión, estructura textual...) (Graham, Harris y Larsen, 2001).

En la revisión, el escritor evalúa el discurso escrito, en comparación con la representación mental del mismo, para corregirlo desde el punto de vista formal, conceptual y funcional (Boscolo, 1988; Scardamalia y Bereiter, 1992). Asimismo, la revisión incluye dos subprocesos: a) análisis del texto escrito, en función de los objetivos planteados en el proceso de la planificación y de la coherencia del contenido expresado, según el esquema retórico; b) corrección del texto escrito, de acuerdo con el análisis (Salvador, 1997).

Los procesos de planificación, transcripción y revisión se desarrollan bajo la supervisión y regulación de las habilidades metacognitivas, siendo éstas las siguientes: conocimiento del proceso de escritura y de la estructura textual; conocimiento y 
evaluación de sus propias capacidades y autorregulación del conocimiento y uso de estrategias escritoras necesarias; actitud ante la escritura (Graham y Harris, 1992). Para ello, es necesario, ante todo, motivar al alumnado a participar en múltiples y ricas actividades que le ofrezcan la posibilidad de reflexionar sobre los procesos cognitivos y metacognitivos implicados en la composición escrita, de modo que ésta tenga siempre una intención comunicativa desde la construcción de significados compartida con otros individuos (Camps, 2004; Graham, 2008).

La composición escrita de alumnos con discapacidad auditiva ha sido, sin duda, menos investigada en comparación con los estudios realizados en sujetos oyentes (De la Paz, Swanson y Graham, 1998; Graham, 1997; Graham y Harris, 1999, 2000; Graham, Schwartz y MacArthur, 1993; Harris, Graham y Mason, 2002; Newcomer y Barenbaum, 1991; Scardamalia y Bereiter, 1986). Las investigaciones revisadas ponen de manifiesto ciertas limitaciones por parte del alumnado sordo en el desarrollo de las competencias escritoras. No obstante, hay que señalar que la mayoría de estos estudios se han limitado al análisis de las características de los textos escritos, referidas al uso del vocabulario, la estructura morfosintáctica y la semántica.

Posteriormente, hacia la década de los '90 y, sobre todo, en el ámbito internacional surgen nuevos estudios centrados en las fases y operaciones cognitivas que se activan en la tarea escritora (Arfè y Perondi, 2008; Fabbretti, Volterra y Pontecorvo, 1998; Fabbretti y Tomasuolo, 2006; Gormley y Sarachan-Deily, 1987; Mayer, 2010; Teruggi, 2001, 2003; Van Beijsterveldt y Van Hell, 2008).

En contraste con éstos, en nuestro país los estudios realizados sobre los procesos escritores son muy escasos (Bellés y Teberosky, 1989; Cambra, 1993; Carrillo y Domínguez, 2010; Pertusa y Fernández, 1999; Ramspott, 1991). Como síntesis de algunos resultados encontrados en los últimos años (Gutiérrez, 2006, 2012; Gutiérrez y Salvador, 2006) se puede señalar que la mayoría de los alumnos sordos encuentran dificultades en el desarrollo de los procesos cognitivos y metacognitivos implicados en la composición de textos escritos.

Siguiendo esta línea, en el marco de un proyecto de investigación ${ }^{1}$ se planteó seguir profundizando en el análisis de los procesos que el alumnado sordo desarrolla en la composición escrita, siendo los objetivos concretos enunciados en forma interrogativa los siguientes:

1. Esta investigación forma parte de un proyecto de investigación desarrollado en el Grupo de Investigación "Diversidad, Discapacidad y Necesidades Educativas Especiales" (HUM-782) y subvencionado por el Plan Propio de la Universidad de Almería. Asimismo, se incluyen resultados parciales tomados de un estudio llevado a cabo durante una estancia de investigación desarrollada en la Facoltà di Scienze della Formazione de la Università Milano-Bicocca (Milán, Italia), la cual ha sido avalada por una financiación concedida por el Ministerio de Educación, mediante el Programa Nacional de Movilidad de Recursos Humanos del Plan Nacional de I-D+i 2008-2011, para la realización de una Estancia de Movilidad en el Extranjero, "José Castillejo". 
RAFAELA GUTIÉRREZ CÁCERES Y ANTONIO LUQUE DE LA ROSA Y OYENTES DE ESPAÑA E ITALIA

- ¿Cuáles son los procesos que los alumnos declaran que realizan cuando escriben?

- ¿Se aprecian diferencias entre los alumnos sordos y los alumnos oyentes?

- ¿Se aprecian diferencias en función del contexto escolar (español-italiano)?

\section{Metodología}

\subsection{Participantes}

En este estudio participaron 84 alumnos, de los que 44 estaban escolarizados en un centro situado en España y 40 en un centro de Italia. En relación con el grupo de alumnado procedente del contexto español, la media general en cuanto a edad es 12,82 años, con una desviación típica de 0,55. En relación con el género, predominan los chicos con un $56,8 \%$ frente al $43,2 \%$ que son chicas. De entre ellos, un total de 6 son sordos cuyos padres son oyentes, siendo 4 de ellos los que presentan una sordera severa y el resto una discapacidad auditiva de grado profundo. Respecto al tipo de prótesis auditivas, 3 sujetos emplean audífonos y el resto un implante coclear. El sistema de comunicación más utilizado en el ámbito familiar se refiere al uso de la lengua oral, excepto en dos casos en que se emplea tanto este sistema como la lengua de signos (véase el Cuadro 1).

En cuanto a la metodología didáctica seguida en este contexto español, con este grupo de alumnado se desarrolla un proyecto centrado en:

- Educar conjuntamente al alumnado sordo y oyente (educación inclusiva) con el fin de conseguir un desarrollo integral de la persona a través de los valores de tolerancia y el respeto a la diversidad.

- Potenciar una educación bilingüe utilizando tanto la lengua castellana como la lengua de signos española para la comprensión del hecho comunicativo.

- Desarrollar actividades de escritura fundamentadas en el constructivismo social, partiendo de un tema concreto con unas pautas dadas por el docente o bien versando sobre un contenido libre que fomente la capacidad creadora en la producción de textos escritos.

En referencia al grupo de alumnado procedente del contexto escolar italiano, la media general en cuanto a edad es de 12,60 años con una desviación típica de 0,29. En cuanto al género, la mayoría son chicas y el 32,44\% son chicos. En este grupo un total de 6 alumnos son sordos que, además, emplean unos audífonos, siendo 3 de ellos los que poseen una sordera profunda, otros 2 una discapacidad auditiva de grado severo y uno con una pérdida leve-moderada. Excepto 4 alumnos, el resto tienen padres que son sordos. El sistema de comunicación más utilizado en el ámbito familiar se refiere a la lengua de signos, salvo en dos casos donde se emplean de manera conjunta las dos lenguas: la lengua oral y la lengua de signos (véase el Cuadro 1). 
RAFAELA GUTIÉRREZ CÁCERES Y ANTONIO LUQUE DE LA ROSA ANÁLISIS DE PROCESOS COGNITIVOS EN LA ESCRITURA DE ALUMNOS SORDOS Y OYENTES DE ESPAÑA E ITALIA

CUADRO 1

Características específicas de los alumnos sordos

\begin{tabular}{|c|c|c|c|c|}
\hline & & $\begin{array}{c}\text { TIPO DE PRÓTESIS } \\
\text { AUDITIVA }\end{array}$ & $\begin{array}{c}\text { ESTATUS } \\
\text { DE LOS PADRES }\end{array}$ & $\begin{array}{c}\text { SISTEMA DE } \\
\text { COMUNICACIÓN }\end{array}$ \\
\hline Contexto español & $\begin{array}{c}\text { Severa }(N: 4) \\
\text { Profunda }(N: 2)\end{array}$ & $\begin{array}{c}\text { Audífonos }(N: 3) \\
\text { Implante coclear } \\
(N: 3)\end{array}$ & Oyentes $(N: 6)$ & $\begin{array}{c}\text { Lengua Oral }(N: 4) \\
\text { Sistema Bilingüe } \\
(N: 2)\end{array}$ \\
\hline Contexto italiano & $\begin{array}{c}\text { Leve-Moderada } \\
(N: 1) \\
\text { Severa }(N: 2) \\
\text { Profunda }(N: 3)\end{array}$ & $\begin{array}{c}\text { Audífonos }(N: 6) \\
\text { Oyentes }(N: 4)\end{array}$ & $\begin{array}{c}\text { Lengua de Signos } \\
(N: 4) \\
\text { Sordos }(N: 2)\end{array}$ \\
$\begin{array}{c}\text { Sistema Bilingüe } \\
(N: 2)\end{array}$ \\
\hline
\end{tabular}

Este centro escolar italiano participa en un proyecto de experimentación bilingüe, una de cuyas principales características es el uso de la lengua de signos y la lengua hablada/escrita situadas al mismo nivel, a través tanto de la presencia de un intérprete que media la comunicación entre sordos y oyentes como de la existencia de situaciones de enseñanza-aprendizaje de la lengua de signos para todo el alumnado. Además, desde el punto de vista metodológico en este centro se promueven prácticas educativas de lectura y escritura desde una perspectiva socioconstructivista, centradas tanto en la producción y comprensión de textos en situaciones auténticas de comunicación, como en la reflexión sobre las estrategias cognitivas subyacentes a dichos procesos.

\subsection{Procedimiento de recogida y análisis de datos}

En el proceso de recogida de datos se ha aplicado una adaptación a partir de los cuestionarios relacionados con la autoevaluación sobre los procesos cognitivos y metacognitivos implicados en la escritura, que han sido extraídos de Cisotto (1998), tomando las aportaciones tanto de Harris y Graham $(1992,1996)$ como de Boscolo (1990). De otra parte, este cuestionario (véase Cuadro 2) se ha adaptado en cuanto a su estructura lingüística de acuerdo con las características y necesidades concretas del alumnado sordo, siendo su estimación numérica comprendida entre 4 puntuaciones o valores (1: Nunca; 2: Algunas veces; 3: Frecuentemente; 4: Siempre).

La fiabilidad de este cuestionario se ha calculado a partir de los resultados obtenidos en este estudio utilizando el índice de consistencia interna. Ha resultado ser alta, ya que el coeficiente alfa de Cronbach, en todas las dimensiones, fue superior a 0,65 y próximo a 0,80 (valor alfa $=0,785$ ). El análisis de los enunciados ha mostrado individualmente que son preguntas capaces de discriminar entre las distintas opiniones de los encuestados (desviaciones típicas altas) y medias relativamente centradas en la escala (de 1 a 5 puntos). 
CUADRO 2

Cuestionario sobre procesos cognitivos y metacognitivos en la escritura

\begin{tabular}{|c|c|c|c|c|}
\hline $\begin{array}{l}\text { 1. Cuando escribo un texto, me pongo a escribir enseguida las } \\
\text { ideas que se me vienen a la mente, lo que se me va ocurriendo }\end{array}$ & 1 & 2 & 3 & 4 \\
\hline 2. Hago correcciones y me fijo en la puntuación & 1 & 2 & 3 & 4 \\
\hline $\begin{array}{l}\text { 3. Antes de escribir un texto, me preparo un plan mental del } \\
\text { texto organizando las ideas en un esquema para luego escribir }\end{array}$ & 1 & 2 & 3 & 4 \\
\hline 4. Cuando estoy nervioso o enfadado, mi texto no me sale bien & 1 & 2 & 3 & 4 \\
\hline $\begin{array}{l}\text { 5. Cuando escribo un texto, lo escribo de manera que resulte } \\
\text { claro y que otra persona que lo lea lo entienda }\end{array}$ & 1 & 2 & 3 & 4 \\
\hline 6. Corrijo los errores de ortografía & 1 & 2 & 3 & 4 \\
\hline $\begin{array}{l}\text { 7. Antes de escribir, pienso en cómo ordenar y relacionar las } \\
\text { partes del texto }\end{array}$ & 1 & 2 & 3 & 4 \\
\hline $\begin{array}{l}\text { 8. Cuando escribo un texto, intento y procuro tener claros los } \\
\text { objetivos y las finalidades de la escritura }\end{array}$ & 1 & 2 & 3 & 4 \\
\hline 9. Corrijo si he escrito bien las letras y con buena caligrafía & 1 & 2 & 3 & 4 \\
\hline $\begin{array}{l}\text { 10. Antes de escribir, busco y recojo las ideas en otros materiales } \\
\text { (libro, película...) para escribir un texto realmente bueno }\end{array}$ & 1 & 2 & 3 & 4 \\
\hline $\begin{array}{l}\text { 11. Cuando escribo, intento y trato de encontrar palabras y } \\
\text { expresiones nuevas }\end{array}$ & 1 & 2 & 3 & 4 \\
\hline $\begin{array}{l}\text { 12. Antes de escribir, pienso en el tipo de texto que voy a escribir } \\
\text { (narración, descripción...) }\end{array}$ & 1 & 2 & 3 & 4 \\
\hline $\begin{array}{l}\text { 13. Si alguien me dice que el texto no está bien escrito y que } \\
\text { tengo que mejorarlo, me siento mal y pienso que no aprenderé } \\
\text { nunca a escribir bien }\end{array}$ & 1 & 2 & 3 & 4 \\
\hline 14. Cuando corrijo, cambio sobre todo las palabras & 1 & 2 & 3 & 4 \\
\hline $\begin{array}{l}\text { 15. Cuando escribo, escribo el texto en un borrador, en una hoja } \\
\text { aparte y diferente a la usada para la redacción }\end{array}$ & 1 & 2 & 3 & 4 \\
\hline $\begin{array}{l}\text { 16. Cuando escribo y se me olvidan algunas palabras, consulto el } \\
\text { diccionario, leo y busco en un libro, pregunto a una persona... }\end{array}$ & 1 & 2 & 3 & 4 \\
\hline 17. Hago correcciones y me fijo en la estructura de las frases & 1 & 2 & 3 & 4 \\
\hline $\begin{array}{l}\text { 18. Si cuando escribo, me doy cuenta de que no estoy escribiendo } \\
\text { bien las ideas, entonces cambio el plan mental de texto inicial }\end{array}$ & 1 & 2 & 3 & 4 \\
\hline $\begin{array}{l}\text { 19. Si escribiendo encuentro algunas dificultadas, me convenzo } \\
\text { y me digo a mí mismo que puedo superarlas }\end{array}$ & 1 & 2 & 3 & 4 \\
\hline 20. Mientras escribo, releo y corrijo los errores que hay en el texto & 1 & 2 & 3 & 4 \\
\hline $\begin{array}{l}\text { 21. Hago correcciones fijándome en la estructura del texto o en } \\
\text { el orden de las partes de un texto y en su relación }\end{array}$ & 1 & 2 & 3 & 4 \\
\hline $\begin{array}{l}\text { 22. Cuando escribo, elijo de entre todas las ideas aquella que } \\
\text { mejor se adecua para explicar el título y para desarrollar el } \\
\text { tema }\end{array}$ & 1 & 2 & 3 & 4 \\
\hline
\end{tabular}


23. Cuando me doy cuenta de que algo no va bien en un texto, pido ayuda a otra persona para corregir el texto (al profesor, a un compañero...)

24. Cuando corrijo, controlo si hay ideas inútiles o incompletas

25. Cuando escribo bien un texto, me siento satisfecho y contento del texto escrito

26. Antes de ponerme a escribir, me concentro en lo que voy a escribir

27. Cuando corrijo, me preocupo sobre todo si a las frases les faltan palabras

28. Antes de escribir, pienso y elijo las palabras y las frases adecuadas para expresar claramente lo que quiero decir

29. Sólo cuando he terminado de escribir el texto, lo corrijo para mejorarlo

\begin{tabular}{|l|l|l|l|}
\hline 1 & 2 & 3 & 4 \\
\hline 1 & 2 & 3 & 4 \\
\hline 1 & 2 & 3 & 4 \\
\hline 1 & 2 & 3 & 4 \\
\hline 1 & 2 & 3 & 4 \\
\hline 1 & 2 & 3 & 4 \\
\hline 1 & 2 & 3 & 4 \\
\hline
\end{tabular}

Con el fin de facilitar el análisis de los resultados obtenidos y atendiendo a los distintos procesos implicados en la composición escrita, previamente descritos en el apartado anterior, se han clasificado los diversos ítems del cuestionario asignándolos en las correspondientes categorías de análisis: planificación, transcripción, revisión y metacognición (véase Cuadro 3).

\section{CUADRO 3}

Asignación de los ítems a sus correspondientes categorías relacionadas con los procesos implicados en la composición escrita

\begin{tabular}{|c|l|l|l|c|}
\hline \multirow{2}{*}{} & \multicolumn{4}{|c|}{ CATEGORÍAS DE ANÁLISIS } \\
\cline { 2 - 5 } & \multicolumn{1}{|c|}{ Planificación } & \multicolumn{1}{|c|}{ Transcripción } & \multicolumn{1}{c|}{ Revisión } & Metacognición \\
\hline \multirow{2}{*}{ ÍtEMS } & $\begin{array}{l}1,3,5,7,8,10, \\
12,15,18,22\end{array}$ & \multirow{2}{*}{$11,16,28$} & $\begin{array}{l}2,6,9,14,17,20, \\
21,23,24,27,29\end{array}$ & $4,13,19,25,26$ \\
\hline
\end{tabular}

En el análisis de los datos obtenidos se han utilizado estadísticos descriptivos tales como la frecuencia, la media y la desviación típica, así como la prueba no paramétrica Chi cuadrado de Pearson con objeto de contrastar la hipótesis (bondad de ajuste entre la distribución teórica y observada con los colectivos sordos/oyentes en ambos contextos y el conjunto de los ítems). Al mismo tiempo, se ha realizado una interpretación cualitativa de los resultados analizando la incidencia del contexto escolar de procedencia en la autoevaluación de los procesos cognitivos y metacognitivos implicados en la escritura. 


\section{Resultados}

En este apartado se exponen los resultados del estudio realizado según cada contexto escolar (español/italiano). Para ello, se ha procedido, en primer lugar, a la presentación general de los datos obtenidos, pasando a continuación a realizar un análisis comparativo entre alumnado sordo y oyente en cada una de las categorías de análisis a que hace referencia esta investigación.

\subsection{Contexto español}

\subsubsection{Análisis descriptivo general}

Con relación a la puntuación media general que ha obtenido el grupo de alumnos españoles, señalar que ésta ha resultado de 2,67 (SD: 1,02), siendo un valor medio-alto en cuanto a la realización de las estrategias cognitivas y metacognitivas relacionadas con la adecuada composición de textos escritos (planificación, transcripción, revisión y metacognición).

En este sentido, son el ítem 13 ( $M$ : 3,30), relacionado con la idea de control del proceso de revisión y posibilidad de mejora en la escritura, y el ítem 25 ( $M$ : 3,32 ), referido a la satisfacción ante la corrección de la composición escrita, los que muestran una mayor puntuación, siendo destacable el hecho de que ambos pertenezcan al proceso de la metacognición. Así, el 81,8\% del alumnado afirma que si alguien le dice que el texto no está bien escrito y que tiene que mejorarlo reacciona y controla el proceso de revisión y sus posibilidades de mejora (ítem 13). Además, se puede señalar cómo el 63,6\% de los alumnos afirma con rotundidad que siempre que escribe bien se siente satisfecho y contento (ítem 25).

Por el contrario, las puntuaciones inferiores se recogen en los ítems 14 y 29 ( $M$ : 1,98), advirtiendo que el $75 \%$ del alumnado encuestado opina que en pocas ocasiones los procesos de corrección hacen referencia, sobre todo, a las palabras y el 70,5\% expresa la importancia de corregir un texto durante el proceso de escritura en lugar de hacerlo sólo a su finalización. Al respecto, cabe señalar que ambos ítems se encuadran en el proceso de revisión de la escritura que se ha comentado con anterioridad.

\subsubsection{Análisis comparativo según categorías de análisis}

A continuación se muestra el análisis diferencial entre alumnado sordo y oyente en función de la agrupación categorial de los ítems según la asignación comentada en relación con los procesos implicados en la composición escrita.

\subsubsection{Planificación}

Con relación a esta categoría, se puede comprobar que la puntuación general promedio de ambos colectivos es similar (sordos- $M$ : 2,51; oyentes- $M$ : 2,64; siendo la desviación típica de 0,87 y 1,03, respectivamente), pudiendo inferir que dicho 
proceso se realiza sin distinción en función de la discapacidad mencionada. No obstante, destaca en el colectivo de alumnos sordos la puntuación de los ítems 8 $(M: 3,00)$ y $12(M: 3,17)$, apreciándose, por parte de la totalidad de los encuestados, que cuando escriben un texto intentan (en mayor o menor medida) y procuran tener claros los objetivos y las finalidades de la escritura y que, antes de escribir, piensan en el tipo de texto que van a escribir (narración, descripción, etc.), superando en puntación en esta dimensión al colectivo de alumnos oyentes.

TABLA 1

Porcentajes de respuesta, media y desviación típica en relación con cada ítem del cuestionario, según el alumnado sordo y oyente procedente del contexto español

\begin{tabular}{|c|c|c|c|c|c|c|c|c|c|c|c|c|}
\hline \multirow{2}{*}{ ÍTEMS } & \multicolumn{6}{|c|}{ SORDOS } & \multicolumn{6}{|c|}{ OYENTES } \\
\hline & 1 & 2 & 3 & 4 & M & SD & 1 & 2 & 3 & 4 & M & SD \\
\hline $\begin{array}{c}1 \\
\text { (recodif.) } \\
\end{array}$ & 0,0 & 66,7 & 33,3 & 0,0 & 2,33 & 0,52 & 34,2 & 28,9 & 23,7 & 13,2 & 2,16 & 1,05 \\
\hline 2 & 0,0 & 33,3 & 33,3 & 33,3 & 3,00 & 0,89 & 18,4 & 21,1 & 42,1 & 18,4 & 2,60 & 1,00 \\
\hline 3 & 16,7 & 33,3 & 33,3 & 16,7 & 2,50 & 1,05 & 34,2 & 36,8 & 21,1 & 7,9 & 2,03 & 0,94 \\
\hline $\begin{array}{c}4 \\
\text { (recodif.) } \\
\end{array}$ & 33,3 & 33,3 & 16,7 & 16,7 & 2,17 & 1,17 & 28,9 & 10,5 & 39,5 & 21,1 & 2,52 & 1,13 \\
\hline 5 & 0,0 & 33,3 & 50,0 & 16,7 & 2,83 & 0,75 & 5,3 & 18,4 & 28,9 & 47,4 & 3,18 & 0,93 \\
\hline 6 & 0,0 & 33,3 & 0,0 & 66,7 & 3,33 & 1,03 & 10,5 & 28,9 & 36,8 & 23,7 & 2,74 & 0,95 \\
\hline 7 & 16,7 & 33,3 & 33,3 & 16,7 & 2,50 & 1,05 & 7,9 & 26,3 & 44,7 & 21,1 & 2,79 & 0,87 \\
\hline 8 & 0,0 & 16,7 & 66,7 & 16,7 & 3,00 & 0,63 & 5,4 & 27,0 & 37,8 & 29,7 & 2,92 & 0,89 \\
\hline 9 & 0,0 & 16,7 & 33,3 & 50,0 & 3,33 & 0,82 & 15,8 & 34,2 & 23,7 & 26,3 & 2,61 & 1,05 \\
\hline 10 & 33,3 & 50,0 & 0,0 & 16,7 & 2,00 & 1,10 & 26,3 & 21,1 & 31,6 & 21,1 & 2,47 & 1,11 \\
\hline 11 & 0,0 & 16,7 & 66,7 & 16,7 & 3,00 & 0,64 & 23,7 & 28,9 & 21,1 & 26,3 & 2,50 & 1,13 \\
\hline 12 & 0,0 & 33,3 & 16,7 & 50,0 & 3,17 & 0,99 & 18,4 & 26,3 & 13,2 & 42,1 & 2,79 & 1,19 \\
\hline $\begin{array}{c}13 \\
\text { (recodif.) }\end{array}$ & 16,7 & 16,7 & 33,3 & 33,3 & 2,83 & 1,17 & 7,9 & 7,9 & 23,7 & 60,5 & 3,37 & 0,94 \\
\hline 14 & 0,0 & 66,7 & 16,7 & 16,7 & 2,50 & 0,84 & 42,1 & 34,2 & 15,8 & 7,9 & 1,89 & 0,95 \\
\hline 15 & 33,3 & 50,0 & 0,0 & 16,7 & 2,00 & 1,10 & 23,7 & 28,9 & 21,1 & 26,3 & 2,50 & 1,13 \\
\hline 16 & 33,3 & 33,3 & 16,7 & 16,7 & 2,17 & 1,17 & 28,9 & 23,7 & 21,1 & 26,3 & 2,45 & 1,18 \\
\hline 17 & 0,0 & 33,3 & 50,0 & 16,7 & 2,83 & 0,75 & 8,3 & 25,0 & 38,9 & 27,8 & 2,86 & 0,93 \\
\hline 18 & 16,7 & 50,0 & 33,3 & 0,0 & 2,17 & 0,75 & 18,4 & 31,6 & 18,4 & 31,6 & 2,63 & 1,13 \\
\hline 19 & 16,7 & 16,7 & 16,7 & 50,0 & 3,00 & 1,26 & 15,8 & 15,8 & 21,1 & 47,4 & 3,00 & 1,14 \\
\hline 20 & 0,0 & 50,0 & 16,7 & 33,3 & 2,83 & 0,98 & 24,3 & 24,3 & 32,4 & 18,9 & 2,46 & 1,07 \\
\hline 21 & 33,3 & 0,0 & 66,7 & 0,0 & 2,33 & 1,03 & 18,9 & 21,6 & 43,2 & 16,2 & 2,57 & 0,99 \\
\hline 22 & 0,0 & 50,0 & 33,3 & 16,7 & 2,67 & 0,82 & 8,1 & 27,0 & 24,3 & 40,5 & 2,97 & 1,01 \\
\hline 23 & 0,0 & 33,3 & 50,0 & 16,7 & 2,83 & 0,75 & 31,6 & 28,9 & 13,2 & 26,3 & 2,34 & 1,19 \\
\hline 24 & 0,0 & 33,3 & 33,3 & 33,3 & 3,00 & 0,89 & 7,9 & 28,9 & 26,3 & 36,8 & 2,92 & 1,00 \\
\hline 25 & 33,3 & 0,0 & 0,0 & 66,7 & 3,00 & 1,55 & 7,9 & 10,5 & 18,4 & 63,2 & 3,37 & 0,97 \\
\hline
\end{tabular}


RAFAELA GUTIÉRREZ CÁCERES Y ANTONIO LUQUE DE LA ROSA

ANÁLISIS DE PROCESOS COGNITIVOS EN LA ESCRITURA DE ALUMNOS SORDOS Y OYENTES DE ESPAÑA E ITALIA

\begin{tabular}{|c|c|c|c|c|c|c|c|c|c|c|c|c|}
\hline \multirow{2}{*}{ ÍTEMS } & \multicolumn{1}{|c|}{ SORDOS } & \multicolumn{1}{c|}{ OYENTES } \\
\cline { 2 - 13 } & 1 & 2 & 3 & 4 & $\mathrm{M}$ & $\mathrm{SD}$ & 1 & 2 & 3 & 4 & $\mathrm{M}$ & $\mathrm{SD}$ \\
\hline 26 & 0,0 & 16,7 & 16,7 & 66,7 & 3,50 & 0,84 & 18,4 & 7,9 & 31,6 & 42,1 & 2,97 & 1,13 \\
\hline 27 & 16,7 & 0,0 & 50,0 & 33,3 & 3,00 & 1,10 & 13,2 & 15,8 & 44,7 & 26,3 & 2,84 & 0,97 \\
\hline 28 & 0,0 & 33,3 & 33,3 & 33,3 & 3,00 & 0,89 & 13,2 & 28,9 & 31,6 & 26,3 & 2,71 & 1,01 \\
\hline $\begin{array}{c}29 \\
\text { (recodif.) }\end{array}$ & 50,0 & 33,3 & 16,7 & 0,0 & 1,67 & 0,82 & 36,8 & 31,6 & 23,7 & 7,9 & 2,03 & 0,97 \\
\hline
\end{tabular}

En cambio, el alumnado oyente (76,3\%) destaca en el ítem 5 ( $M: 3,18)$, relacionado con la intención de escribir un texto de manera que resulte claro en su conjunto y que otra persona que lo lea lo entienda. En este sentido, mientras que en el colectivo de alumnos sordos sólo un 16,7\% responde que siempre realiza dicho proceso relacionado con la planificación, en los alumnos oyentes dicha afirmación se da en el 47,4\% de los encuestados para la totalidad de las composiciones escritas.

\subsubsection{Transcripción}

Con relación a dicho proceso y su relación con los aspectos sintácticos, léxicos o grafomotores -con la consiguiente puesta en funcionamiento del conocimiento lingüístico y de las reglas gramaticales-, se aprecia una puntuación promedio más elevada en el alumnado sordo $(M: 2,72 ; S D$ : 0,90$)$ frente al oyente $(M: 2,55 ; S D$ : 1,11), destacando dicho colectivo en los ítems 11 y 28, en los cuales todos los encuestados afirman que, en mayor o menor medida, cuando escriben, intentan encontrar palabras y expresiones nuevas, pensando y eligiendo las palabras y las frases adecuadas para expresar lo que quieren decir.

\subsubsection{Revisión}

Conceptualizando la revisión como el proceso cognitivo en el que la persona analiza y corrige el discurso escrito comparándolo con la representación mental del mismo, tanto desde el punto de vista formal como conceptual, a la vista de los resultados obtenidos se puede observar cómo la puntuación del alumnado sordo es sensiblemente superior a la encontrada en el grupo de alumnado oyente ( $M$ : 2,79 frente a 2,53; SD: 0,90 y 1,01, respectivamente), destacando en aquel colectivo los resultados obtenidos en los ítems 6 y 9, en los cuales la totalidad del alumnado sordo manifiesta corregir, en mayor o menor medida, los errores de ortografía y caligrafía, frente a un 10,5\% y un 15,8\% -respectivamente-, de alumnado oyente que manifiesta abiertamente no corregirlos nunca.

Por el contrario, cabe reseñar que, a pesar de no mostrar una puntuación elevada por parte de ninguno de los grupos en el ítem 29 relacionado con la corrección del texto durante el proceso de realización $-\mathrm{y}$ no sólo al final-, el colectivo de oyentes manifiesta una puntuación superior en dicho aspecto existiendo un 50\% de alumnado sordo que no lo realiza nunca y ninguno que manifieste que lo realice siempre (prueba Chi-cuadrado de Pearson: 0,004). 


\subsubsection{Metacognición}

En cuanto a los procesos metacognitivos del proceso de escritura, cabe señalar que en este aspecto resulta ser el colectivo de alumnado oyente el que aventaja sensiblemente en los resultados generales de dicha categoría ( $M$ : 3,05 frente a 2,90; SD: 1,06 y 1,20, respectivamente), destacando la puntuación obtenida en los ítems 13 y 25 ( $M$ : 3,37 en ambos). En este sentido, el 84,2\% del alumnado oyente afirma que siempre o frecuentemente que alguien le critica sus elaboraciones escritas considera que puede mejorarlas y llegar a hacerlas con corrección y el 81,6\% de dicho grupo manifiesta su satisfacción cuando realiza bien un texto escrito.

No obstante, merece comentarse la alta puntuación obtenida por el alumnado sordo en el ítem 26, al reseñarse por parte de la totalidad de los encuestados que, en mayor o menor medida, antes de ponerse a escribir se concentra en lo que va a realizar.

\subsection{Contexto italiano}

\subsubsection{Análisis descriptivo general}

Con respecto a la puntuación media general se ha obtenido un promedio de 2,53, que se encuentra comprendido entre el valor 2 correspondiente a "algunas veces" y el valor 3 "frecuentemente" ( $S D$ : 0,92), lo cual indica que el alumnado procedente del contexto italiano opina que realiza, unos en ocasiones y otros con frecuencia, las estrategias relacionadas con cada uno de los procesos implicados en la expresión escrita, que son: planificación, transcripción, revisión y metacognición.

Se puede destacar cómo en el conjunto de los ítems son el 5 y el 26 los que especialmente destacan superando el valor promedio $(M: 3,40)$. En este sentido, el $82,5 \%$ del alumnado afirma que cuando escribe lo hace de manera que resulte claro intentando la futura comprensión por parte del destinatario (ítem 5). Asimismo, el $87,5 \%$ coincide en señalar en que se concentra en el contenido de su escritura (ítem 26). De otra parte, se ha encontrado que es el ítem 10 el más destacado negativamente ( $M: 1,62)$, de modo que el $55 \%$ considera que nunca realiza la estrategia referida a la búsqueda y selección de fuentes de información, necesaria para desarrollar el proceso de planificación.

\subsubsection{Análisis comparativo según categorías de análisis}

A continuación se muestra el análisis diferencial entre alumnos sordos y oyentes en función de la clasificación de los ítems según las categorías referidas a los procesos implicados en la composición escrita. 
RAFAELA GUTIÉRREZ CÁCERES Y ANTONIO LUQUE DE LA ROSA

ANÁLISIS DE PROCESOS COGNITIVOS EN LA ESCRITURA DE ALUMNOS SORDOS Y OYENTES DE ESPAÑA E ITALIA

TABLA 2

Porcentajes de respuesta, media y desviación típica en relación con cada ítem del cuestionario, según el alumnado sordo y oyente procedente del contexto italiano

\begin{tabular}{|c|c|c|c|c|c|c|c|c|c|c|c|c|}
\hline \multirow{2}{*}{ ÍTEMS } & \multicolumn{6}{|c|}{ SORDOS } & \multicolumn{6}{|c|}{ OYENTES } \\
\hline & 1 & 2 & 3 & 4 & $M$ & SD & 1 & 2 & 3 & 4 & $\mathrm{M}$ & $\mathrm{SD}$ \\
\hline $\begin{array}{c}1 \\
\text { (recodif.) }\end{array}$ & 16,7 & 16,7 & 66,7 & 0 & 2,50 & 0,84 & 20,6 & 26,5 & 44,1 & 8,8 & 2,41 & 0,92 \\
\hline 2 & 0 & 33,3 & 50 & 16,7 & 2,83 & 0,75 & 5,9 & 52,9 & 26,5 & 14,7 & 2,50 & 0,82 \\
\hline 3 & 50 & 33,3 & 0 & 16,7 & 1,83 & 1,17 & 41,2 & 29,4 & 20,6 & 8,8 & 1,97 & 1,00 \\
\hline $\begin{array}{c}4 \\
\text { (recodif.) }\end{array}$ & 50 & 16,7 & 16,7 & 16,7 & 2,00 & 1,26 & 35,3 & 17,6 & 35,3 & 11,8 & 2,23 & 1,07 \\
\hline 5 & - & 0 & 33,3 & 66,7 & 3,67 & 0,52 & - & 20,6 & 23,5 & 55,9 & 3,35 & 0,81 \\
\hline 6 & 0 & 16,7 & 50 & 33,3 & 3,17 & 0,75 & 5,9 & 14,7 & 38,2 & 41,2 & 3,15 & 0,89 \\
\hline 7 & 0 & 33,3 & 50 & 16,7 & 2,83 & 0,75 & 14,7 & 20,6 & 32,4 & 32,4 & 2,82 & 1,06 \\
\hline 8 & 16,7 & 16,7 & 50 & 16,7 & 2,67 & 1,03 & 27,3 & 36,4 & 30,3 & 6,1 & 2,15 & 0,90 \\
\hline 9 & 16,7 & 16,7 & 16,7 & 50 & 3,00 & 1,26 & 26,5 & 20,6 & 32,4 & 20,6 & 2,47 & 1,11 \\
\hline 10 & 66,7 & 16,7 & 0 & 16,7 & 1,67 & 1,21 & 52,9 & 32,4 & 14,7 & 0 & 1,62 & 0,74 \\
\hline 11 & 16,7 & 33,3 & 16,7 & 33,3 & 2,67 & 1,21 & 11,8 & 47,1 & 20,6 & 20,6 & 2,50 & 0,96 \\
\hline 12 & 16,7 & 16,7 & 33,3 & 33,3 & 2,83 & 1,17 & 5,9 & 29,4 & 32,4 & 32,4 & 2,91 & 0,93 \\
\hline $\begin{array}{c}13 \\
\text { (recodif.) }\end{array}$ & 33,3 & 0 & 16,7 & 50 & 2,83 & 1,47 & 8,8 & 14,7 & 32,4 & 44,1 & 3,12 & 0,98 \\
\hline 14 & 0 & 66,7 & 16,7 & 16,7 & 2,50 & 0,84 & 11,8 & 55,9 & 26,5 & 5,9 & 2,26 & 0,75 \\
\hline 15 & 66,7 & 16,7 & 16,7 & 0 & 1,50 & 0,84 & 44,1 & 23,5 & 20,6 & 11,8 & 2,00 & 1,07 \\
\hline 16 & 33,3 & 16,7 & 0 & 50 & 2,67 & 1,50 & 26,5 & 47,1 & 20,6 & 5,9 & 2,06 & 0,85 \\
\hline 17 & 33,3 & 50 & 16,7 & 0 & 1,83 & 0,75 & 20,6 & 47,1 & 26,5 & 5,9 & 2,18 & 0,83 \\
\hline 18 & 50 & 33,3 & 16,7 & - & 1,67 & 0,82 & 35,3 & 47,1 & 17,6 & - & 1,82 & 0,72 \\
\hline 19 & 0 & 16,7 & 50 & 33,3 & 3,17 & 0,75 & 11,8 & 23,5 & 47,1 & 17,6 & 2,70 & 0,90 \\
\hline 20 & 0 & 33,3 & 16,7 & 50 & 3,17 & 0,98 & 6,1 & 21,2 & 36,4 & 36,4 & 3,03 & 0,92 \\
\hline 21 & 33,3 & 16,7 & 50 & 0 & 2,17 & 0,98 & 14,7 & 32,4 & 41,2 & 11,8 & 2,50 & 0,90 \\
\hline 22 & 16,7 & 33,3 & 33,3 & 16,7 & 2,50 & 1,05 & 11,8 & 26,5 & 29,4 & 32,4 & 2,82 & 1,03 \\
\hline 23 & 16,7 & 33,3 & 16,7 & 33,3 & 2,67 & 1,21 & 26,5 & 29,4 & 32,4 & 11,8 & 2,29 & 1,00 \\
\hline 24 & 0 & 16,7 & 83,3 & 0 & 2,83 & 0,41 & 8,8 & 41,2 & 38,2 & 11,8 & 2,53 & 0,82 \\
\hline 25 & 0 & 0 & 16,7 & 83,3 & 3,83 & 0,41 & 8,8 & 20,6 & 23,5 & 47,1 & 3,09 & 1,02 \\
\hline 26 & 0 & 0 & 50 & 50 & 3,50 & 0,55 & 5,9 & 8,8 & 26,5 & 58,8 & 3,38 & 0,89 \\
\hline 27 & 16,7 & 50 & 16,7 & 16,7 & 2,33 & 1,03 & 11,8 & 47,1 & 29,4 & 11,8 & 2,41 & 0,86 \\
\hline 28 & 0 & 33,3 & 16,7 & 50 & 3,17 & 0,98 & 8,8 & 35,3 & 41,2 & 14,7 & 2,62 & 0,85 \\
\hline $\begin{array}{c}29 \\
\text { (recodif.) }\end{array}$ & 33,3 & 16,7 & 33,3 & 16,7 & 2,33 & 1,21 & 35,3 & 23,5 & 32,4 & 8,8 & 2,15 & 1,02 \\
\hline
\end{tabular}




\subsubsection{Planificación}

En relación con el proceso de planificación, se ha obtenido un promedio de 2,37 en el caso de alumnos sordos, mientras que en el de los oyentes es de 2,39 (SD: 0,94 y 0,92 , respectivamente). No obstante, se ha encontrado que existen discrepancias entre ambos colectivos, en relación con lo enunciado en los ítems 8 y 15.

Así el $66,7 \%$ de alumnos sordos afirma que cuando escribe pretende tener claros los objetivos y finalidades de la escritura (ítem 8), mientras que el 63,7\% de alumnos oyentes considera que esta actividad cognitiva no la desarrolla.

Un dato significativo a destacar es cómo el 83,4\% de alumnos sordos no utiliza ningún borrador para escribir. Del mismo que sus compañeros oyentes, aunque en un porcentaje inferior $(67,6 \%)$.

\subsubsection{Transcripción}

Referente al proceso de transcripción el valor promedio es de 2,46, siendo superior la puntuación media general correspondiente al ítem 28, donde el $66,7 \%$ de los alumnos sordos y el 55,9\% de los alumnos oyentes considera que piensa y selecciona aquellas palabras y frases para escribir sus ideas en base a la comunicación adecuada de las mismas. Asimismo, se puede destacar cómo el alumnado sordo aventaja aunque ligeramente en la puntuación media con respecto a sus compañeros oyentes ( $M: 2,84>2,39)$, destacando en el ítem 16 (prueba Chi-cuadrado de Pearson: 0,007). En efecto, el 50\% de los alumnos sordos afirma que utiliza diversas fuentes de información para la búsqueda de palabras adecuadas para escribir textos de calidad (item 16), mientras que en el caso de sus compañeros oyentes sólo lo hace un 26,5\%.

\subsubsection{Revisión}

Con respecto al proceso de revisión se ha obtenido un promedio de 2,51, siendo superior el valor medio correspondiente al ítem 6, donde entre el 83,3\% de los alumnos sordos y el $79,4 \%$ de los oyentes afirman que corrigen los errores de ortografía. Además, se puede destacar cómo el 66,7\% de los alumnos sordos y el $72,8 \%$ de los oyentes señalan que durante la escritura releen el texto y corrigen los errores existentes (ítem 20).

Asimismo, es interesante señalar cómo el 66,7\% del alumnado sordo considera que corrige atendiendo a la caligrafía, frente al 53\% de sus compañeros oyentes (ítem 9). Asimismo, se puede observar cómo el 32,4\% de los alumnos oyentes afirma que revisa fijándose en la gramática, mientras que el 83,3\% del alumnado sordo mantiene que no desarrolla esta habilidad cognitiva (item 17; prueba Chicuadrado de Pearson: 0,005). Así como también hay que señalar cómo el 50\% de alumnos sordos está de acuerdo con la idea de que recurre a otra persona para la revisión de sus textos, frente al 44,2\% de sus compañeros oyentes (ítem 23).

Particularmente significativo se ha encontrado en relación con el ítem 2, donde el $66,7 \%$ de los alumnos sordos opina que cuando revisa se fija en la puntuación, 
frente al 41,2\% de sus compañeros oyentes. Así como también en referencia al ítem 24 , ya que mientras el $83,3 \%$ del alumnado sordo considera que revisa si existen ideas inútiles o incompletas, sólo es el 50\% de los compañeros oyentes quien afirma que esta habilidad cognitiva la realiza.

\subsubsection{Metacognición}

En cuanto al proceso referido a la metacognición, se ha obtenido un promedio de 2,93, un valor superior al promedio encontrado en las restantes categorías de este análisis. Se puede destacar cómo es el ítem 26 el mejor valorado positivamente. Así, la totalidad del alumnado sordo así como el 85,3\% del alumnado oyente afirman que se concentran en lo que van a escribir. Además, el 100\% de los alumnos sordos considera que se siente satisfecho y contento cuando escribe, frente al 70,6\% de sus compañeros oyentes (ítem 25).

Mientras que es el ítem 4 aquel cuya puntuación media destaca negativamente en el conjunto de los ítems relativos a la categoría referida al proceso de metacognición. En este sentido, se puede señalar cómo el 66,7\% de los alumnos sordos y el 52,9\% de los oyentes afirman que, no son capaces de controlar sus sentimientos negativos para poder seguir escribiendo.

Otro dato relevante se ha observado en relación con el ítem 19, donde el 83,3\% del alumnado sordo afirma positivamente que, si encuentra dificultades escritoras, se siente capaz de superarlas, mientras que esta habilidad cognitiva la ha considerado sólo el 64,7\% del alumnado oyente.

\section{DisCUSIÓN Y CONCLUSIONES}

A partir del análisis e interpretación de los resultados obtenidos y atendiendo a los valores descriptivos referidos a la puntuación media general tras la aplicación del cuestionario, se puede concluir en términos generales que los alumnos sordos y oyentes escolarizados en los centros procedentes de España e Italia coinciden en afirmar que realizan las estrategias necesarias para desarrollar los procesos implicados en la composición de textos escritos: planificación, transcripción, revisión y metacognición. En este sentido, se deduce cómo la participación por parte de ambos centros escolares en las experiencias de integración así como en los proyectos bilingües (lengua de signos y lengua mayoritaria, oficiales del país) favorece tanto al alumnado sordo como al oyente en el aprendizaje y mejora de los procesos escritores. No obstante, es preciso señalar la necesidad de llevar a cabo diversas investigaciones que permitan comprender y analizar en profundidad la incidencia del contexto escolar en el aprendizaje de la composición escrita.

En cualquier caso, hay que destacar ante todo que la discapacidad auditiva no es un obstáculo por sí solo para el desarrollo de la composición escrita y, en este caso, para la autoevaluación sobre los procesos cognitivos y metacognitivos. Estos resultados positivos en relación con las habilidades cognitivas dan muestras de esperanzas acerca del colectivo de personas sordas en el ámbito de la expresión escrita, 
RAFAELA GUTIÉRREZ CÁCERES Y ANTONIO LUQUE DE LA ROSA ANÁLISIS DE PROCESOS COGNITIVOS EN LA ESCRITURA DE ALUMNOS SORDOS Y OYENTES DE ESPAÑA E ITALIA

frente a la idea de incapacidad y/o diferencia dada por las dificultades específicas encontradas en otras investigaciones realizadas en relación con la escritura centradas en el proceso (Arfè y Perondi, 2008; Cambra, 1993; Carrillo y Domínguez, 2010; Mayer, 2010). No obstante, ante la escasez de este tipo de estudios es prioritario insistir en la necesidad de realizar investigaciones centradas en el análisis de los procesos cognitivos y metacognitivos de la escritura en alumnos sordos.

En relación con las categorías de análisis a que hace referencia este trabajo, se ha encontrado que en ambos centros escolares tanto el alumnado sordo como el oyente destaca en los ítems relacionados con la metacognición. Al respecto se puede mostrar la posible incidencia de las estrategias metodológicas utilizadas en dichos contextos en relación con el aprendizaje de la escritura, favoreciendo en el alumnado el desarrollo de las concepciones positivas que éste manifiesta acerca de los procesos metacognitivos, con independencia de si presenta o no una discapacidad auditiva.

Igualmente, es de reseñar que el colectivo procedente de España obtiene mayor puntuación en el conjunto de los ítems relativos a la planificación. En concreto, se puede apreciar cómo el 69,8\% de los alumnos escolarizados en el centro español, frente al $41 \%$ de los alumnos procedentes del contexto italiano, afirma que pretende establecer claramente los objetivos y las finalidades de la escritura (ítem 8). Y lo que es más significativo, el 47,8\% del colectivo español considera que busca y recoge ideas en otras fuentes de información para escribir bien, siendo esta estrategia tenida en cuenta sólo por el 15\% del alumnado escolarizado en el centro italiano (ítem 10).

Se ha encontrado también cómo el alumnado procedente del contexto español destaca en sus puntuaciones relativas a la categoría de transcripción y, en especial, en relación con el ítem 18. En efecto, el 47,8\% del alumnado español afirma que, cuando escriben y observan que no progresan, cambian el plan mental inicial, mientras que en el centro italiano sólo el 17,5\% ha considerado la realización de esta estrategia. Asimismo, se ha observado cómo el alumnado español supera en la categoría de revisión excepto en lo relativo al ítem 20. Así, y curiosamente, el $71,8 \%$ del alumnado escolarizado en el centro italiano señala que cuando escriben un texto lo releen para corregir los errores existentes, frente al 51,1\% del alumnado español.

Comparando los resultados obtenidos en función de la clasificación de alumnado según si posee o no discapacidad auditiva, se puede concluir que los alumnos sordos aventajan al grupo de oyentes en la categoría relativa a la transcripción y, en mayor medida, en el caso del centro escolar italiano, sobre todo en relación con el ítem 16 ("Cuando escribo y se me olvidan algunas palabras, consulto el diccionario, leo y busco en un libro, pregunto a una persona..."; $M$ : 2,67 y 2,06 , respectivamente).

Centrándonos en el colectivo de alumnos sordos, se puede destacar cómo los escolarizados en el centro español destacan ligeramente en sus puntuaciones medias en los ítems referidos a las categorías de revisión, sobre todo, en relación 
con los ítems 17 ("Hago correcciones y me fijo en la estructura de las frases"; Sordos españoles- $M$ : 2,83; Sordos italianos- $M$ : 1,83) y 27 ("Cuando corrijo, me preocupo sobre todo si a las frases les faltan palabras"; Sordos españoles-M: 3,00; Sordos italianos-M: 2,33), excepto en lo relativo al ítem 29 ("Sólo cuando he terminado de escribir el texto, lo corrijo para mejorarlo"; Sordos españoles-M: 1,67; Sordos italianos- $M$ : 2,33).

Igualmente, se ha encontrado un ligero incremento en las puntuaciones medias por parte de los alumnos sordos del contexto español en el conjunto de los ítems referidos a la planificación y especialmente en el ítem 3 ("Antes de escribir un texto, me preparo un plan mental del texto organizando las ideas en un esquema para luego escribirn; Sordos españoles- $M$ : 2,50; Sordos italianos- $M$ : 1,83 ), salvo en el ítem 5 superado en su puntuación media por el grupo de alumnado sordo escolarizado en el centro italiano ("Cuando escribo un texto, lo escribo de manera que resulte claro y que otra persona que lo lea lo entienda"; $M$ : 3,67 frente a 2,83). En cambio, es el colectivo de alumnos sordos procedentes de Italia quien destaca en la categoría de transcripción y, sobre todo, en la de metacognición. Especialmente relevante es de señalar el ítem 25 con un promedio de 3,83, frente al 3,00 en el grupo de alumnos sordos procedentes de España ("Cuando escribo bien un texto, me siento satisfecho y contento del texto escrito").

Todos estos datos manifiestan que, si bien como se señala en diversas investigaciones indicadas en el marco teórico (Gutiérrez, 2006, 2012; Gutiérrez y Salvador, 2006), el colectivo de alumnado sordo presenta dificultades para llevar a cabo los diversos procesos de planificación, transcripción, revisión y metacognición en el desarrollo de la lectoescritura, no podemos apreciar que los resultados sean significativamente diferentes a los obtenidos en el colectivo de oyentes, tanto en el contexto español como italiano.

Dicha conclusión se ve matizada por el hecho de que el colectivo sordo del presente estudio aventaja al de oyentes en los procesos de transcripción (plasmación por escrito de las ideas previamente planificadas -uso y conocimiento de la estructura discursiva y la forma sintáctica del texto-) contrastando con las discusiones que manifiestan dichos autores (Gutiérrez, 2006, 2012; Gutiérrez y Salvador, 2006), acerca de la dificultad del colectivo sordo para el desarrollo de estas habilidades.

Este hecho, unido a la variabilidad en las respuestas proporcionadas por cada estatus de sujetos a cada ítem del cuestionario (autoevaluación acerca de los procesos cognitivos y metacognitivos implicados en la composición escrita), hace que se considere necesario el desarrollo de estudios de casos cuyo objetivo sea analizar las concepciones que este tipo de alumnado desarrolla con respecto a la composición escrita así como las prácticas educativas dirigidas a su aprendizaje y mejora. Asimismo, se contempla la importancia de colaborar en el estudio de propuestas que avancen hacia planteamientos educativos que favorezcan la adecuada integración escolar del colectivo de alumnado sordo y la mejora de la composición escrita, pretendiendo continuar en esta línea con posteriores propuestas que complementen lo aquí expuesto. 
RAFAELA GUTIÉRREZ CÁCERES Y ANTONIO LUQUE DE LA ROSA ANÁLISIS DE PROCESOS COGNITIVOS EN LA ESCRITURA DE ALUMNOS SORDOS Y OYENTES DE ESPAÑA E ITALIA

Por último, con objeto de completar el presente estudio, se pasa a continuación a detallar algunos ejemplos de propuestas didácticas que podrían desarrollarse en el aula para mejorar el desarrollo de los procesos cognitivos y metacognitivos en la escritura. Es importante destacar la necesidad de incidir en dichas tareas escritoras de manera colaborativa (familia-escuela), no relegando su tratamiento sólo en el centro ni tampoco dedicando un tiempo específico a las mismas sólo en la clase de lengua, sino también en el resto de materias. Asimismo, se deben desarrollar las actividades escritoras siempre en coherencia con los intereses, aficiones y necesidades del alumnado.

Respecto a la atención al alumnado sordo es conveniente priorizar los aspectos funcionales del lenguaje escrito: comunicación escrita (cartas, chat, periódico escolar), a través de estrategias didácticas que potencien aprendizajes significativos partiendo de lo que los alumnos conocen y relacionando los contenidos con sus intereses. Es pues necesario evitar enfrentar al alumno sordo de entrada a lo que no está a su alcance, debiendo conocer el profesor bien las características del alumno para adaptarse a sus posibilidades.

Se han de planificar experiencias que sirvan de punto de partida para entender las materias, y relacionar la realidad con lo estudiado, utilizando la expresión escrita como instrumento de desarrollo cognitivo, autorregulación y expresión de ideas adquiridas, al tiempo que permita la personalización y creatividad a través del lenguaje.

En casos específicos, pueden ser beneficiosas actuaciones como situar al alumno junto a los que conozcan y utilicen la lengua de signos, sirviendo dicha tutoría entre pares como sistema de coevaluación del proceso escritor. Finalizando, es preciso destacar que la enseñanza en torno a procesos pretende alcanzar la autonomía en el aprendizaje, reforzando el conocimiento y uso de los procedimientos como actividades secuenciadas, guiando al alumnado sordo y oyente en la realización de las actividades escritoras.

\section{REFERENCIAS BIBLIOGRÁFICAS}

Arfè, B. y Perondi, I. (2008). Deaf and hearing students' referential strategies in writing: What referential cohesion tells us about deaf students' literacy development. First Language, 28, 355-374. http://dx.doi.org/10.1177/0142723708091043

Arroyo, R. y Salvador, F. (2009). Research on cognitive, social and cultural processes of written Communications. Cognitive Processing, 10, 263-268. http://dx.doi.org/10.1007/ 810339-009-0255-1

Bellés, R. M. y Teberosky, A. (1989). Producción e interpretación de textos escritos por niños sordos pequeños integrados en escuelas ordinarias. Convocatoria de Proyectos de Investigación de 1985. Madrid: CIDE.

Berninger, V. W.; Fuller, F. y Whitaker, D. (1996). A process model of writing development: Across the life span. Educational Psychology Review, 8, 193-205. http://dx.doi. org/10.1007/BF01464073 
Berninger, V. W. y Whitaker, D. (1993). Theory Based Branching Diagnosis of Writing Disabilities. School Psychology Review, 22 (4), 623-642.

Boscolo, P. (1988). Scrittura e processi cognitivi: alcune riflessioni sui risultati della ricerca e alcune implicazioni per la prattica educativa. Orientamenti Pedagogici, 35 (4), 674-684.

- (Coord.) (1990). Insegnare i processi della scrittura nella scuola elementare. Firenze: la Nuova Italia.

Cambra, C. (1993). Proceso de composición de textos narrativos escritos por alumnos sordos. Revista de Logopedia, Foniatría y Audiología, 13 (2), 73-78. http://dx.doi.org/10.1016 S0214-4603(93)75563-1

Camps, A. (2004). Miradas diversas a la enseñanza y aprendizaje de la composición escrita. Lectura y Vida, 4, 14-23.

Carrillo, M. y Domínguez, A. B. (Coords.) (2010). Dislexia y sordera: líneas actuales en el estudio de la lengua escrita y sus dificultades. Archidona: Aljibe.

Cisotto, L. (1998). Scrittura e metacognizione. Linee teoriche e proposte operative. Trento: Erickson.

De la Paz, S.; Swanson, P. N. y Graham, S. (1998). The Contribution of Executive Control to the Revising by Students with Writing and Learning Difficulties. Journal of Educational Psychology, 90 (3), 448-460. http://dx.doi.org/10.1037/0022-0663.90.3.448

Fabbretti, D. y Tomasuolo, E. (Coords.) (2006). Scrittura e sordità. Roma: Carocci.

Fabbretti, D.; Volterra, V. y Pontecorvo, C. (1998). Written language abilities in deaf Italians. Journal of Deaf Studies and Deaf Education, 3 (3), 231-244. http://dx.doi.org/10.1093 oxfordjournals.deafed.a014353

Fernández, M. P. y Pertusa, E. (2007). Estado del arte sobre la enseñanza de la lengua escrita en el niño sordo. Descargado el 15 de mayo de 2010. http://www.deal-leonardo-eu.

Flower, L. y Hayes, J. R. (1981). A cognitive process theory of writing. College Composition and Communication, 32 (4), 365-387. http://dx.doi.org/10.2307/356600

- (1984). Images, plans and prose: The representation of meaning in writing. Written Communication, 1 (1), 120-160.

Gormley, K. y Sarachan-Deily, B. (1987). Evaluating Hearing-Impaired Students' Writing: A Practical Approach. Volta Review, 89 (3), 157-166.

Graham, S. (1997). Executive control in the revising of students with learning and writing difficulties. Journal of Educational Psychology, 89, 223-234. http://dx.doi. org/10.1037/0022-0663.89.2.223

- (2008). Research on writing development, practice, instruction, and assessment. Reading and Writing, 21, 1-2. http://dx.doi.org/10.1007/s11145-007-9069-7

Graham, S. y Harris, K. B. (1992). Cognitive strategy instruction in written language for learning disabled students. En S. A. Vogel (Ed.). Educational alternatives for students with learning disabilities (pp. 91-116). New York: Springer-Verlag.

- (1999). Assessment and Intervention in Overcoming Writing Difficulties: An Illustration from the Self-Regulated Strategy Development Model. Language, Speech, and Hearing Services in Schools, 30 (3), 255-264.

- (2000). The role of Self-Regulation and Transcription Skills in Writing and Writing Development. Educational Psychologist, 35 (1), 3-12. http://dx.doi.org/10.1207 515326985EP3501_2

Graham, S.; Harris, K. R. y Larsen, L. (2001). Prevention and intervention of writing difficulties for students with learning disabilities. Learning Disabilities: Research and Practice, 16 (2), 74-84. http://dx.doi.org/10.1111/0938-8982.00009 
Graham, S.; Schwartz, S. S. y MacArthur, C. A. (1993). Knowledge of writing and the composing process, attitudes toward writing and self-efficacy for students with and without learning disabilities. Journal of Learning Disabilities, 26 (4), 237-249. http://dx.doi. org/10.1177/002221949302600404

Gutiérrez, R. (2006). La revisión de textos escritos con errores en alumnos sordos de Educación Secundaria. Enseñanza, 24, 281-294.

- (2012). Análisis del proceso de transcripción en la expresión escrita de alumnos sordos. Revista Complutense de Educación, 23, 2, 331-346.

Gutiérrez, R. y Salvador, F. (2006). El proceso de planificación en la expresión escrita de alumnos sordos: estudio de casos en educación secundaria. Revista de Educación, 339, 435-453.

Harris, K. R. y Graham, S. (1992). Self-regulated strategy development: A part of the writing process. En M. Pressley, K. R. Harris y J. T. Guthrie (Eds.). Promoting academic competence and literacy in school (pp. 277-309). San Diego, CA: Academic Press.

- (1996). Making the writing process work: Strategies for composition and self-regulation. Cambridge, Massuchusetts: Brookline Books.

Harris, K. R.; Graham, S. y Mason, L. H. (2002). Developing self-regulated writers. Theory into Practise, 41 (2), 110-115. http://dx.doi.org/10.1207/s15430421tip4102_7

Hayes, J. R. (1996). A new framework for understanding cognition and affect in writing. In C. M. Levy y S. E. Randsell (Eds.). The science of writing: theories, methods, individual differences and applications (pp. 1-27). Mahwah: Lawrence Erlbaum.

Jáudenes, C. (Dir.) (2009). FIAPAS: Estudios sobre población con sordera en España. FIAPAS, 130. Monográfico.

MacArthur, C. A.; Graham, S. y Fitzgerald, J. (Eds.) (2006). Handbook of writing research. New York: Guilford Press.

Mayer, C. (2010). The demands of writing and the deaf writer. En M. Marshark y P. E. Spencer (Eds.). The Oxford Handbook of Deaf Studies, Language, and Education, vol. 2 (pp. 144-155). New York: Oxford University Press.

Newcomer, P. y Barenbaum, E. (1991). The written composition ability of children with learning disabilities. A review of the literature. Journal of Learning Disabilities, 24 (10), 578-593. http://dx.doi.org/10.1177/002221949102401001

Pertusa, E. y Fernández, M. P. (1999). Representación fonológica, aprendizaje de la escritura y alumnos sordos. Revista de Logopedia, Foniatria y Audiología, 19 (1), 2-10. http:// dx.doi.org/10.1016/S0214-4603(99)75696-2

Ramspott, A. (1991). La comprensión y la producción de cuentos en niños y adolescentes sordos. Tesis Doctoral. Barcelona: Universidad Autónoma de Barcelona.

Salvador, F. (1997). Dificultades en el aprendizaje de la expresión escrita. Archidona: Aljibe.

Scardamalia, M. y Bereiter, C. (1986). Research on written composition. En C. M. Wittrock (Ed.). Handbook of research on teaching (pp. 778-803). Nueva York: McMillan. 3. ․ edición.

- (1992). Dos modelos explicativos de los modelos de composición escrita. Infancia y Aprendizaje, 58, 43-64.

Teruggi, L. A. (2001). Pratiche di lectura e di scrittura nei bambini sordi. Età Evolutiva, 68, 81-86.

- (2003). Il processo di concettualizzazione della lengua scritta nei bambini sordi. En L. A. Teruggi (Ed.). Una scuola, due lingue. L'esperienza di bilingüismo della scuola dell'Infanzia ed Elementare di Cossato (pp. 313-333). Milano: Franco Angeli.

Van Beijsterveldt, L. M. y Van Hell, J. G. (2008). Evaluative expression in deaf children's written narratives. International Journal of Language and Communication Disorders, First Article, 1-18. 